\title{
The Measurement of Dilational Properties of Liquid Surfaces
}

\author{
A. F. M. SNIK, W. J. J. KOUIJZER, J. C. M. KELTJENS, AND Z. HOUKES* \\ Department of Physics, Eindhoven University of Technology, P.O. Box 513, Eindhoven, the Netherlands, and \\ *Department of Electrical Engineering, Twente University of Technology, Enschede, the Netherlands
}

Received March 22, 1982; accepted October 8, 1982

\begin{abstract}
For measurements of viscoelastic properties of monolayer covered interfaces, the longitudinal wave technique is used. The longitudinal wave is generated in the plane of the interface, using a horizontal oscillating barrier. The wave propagation depends on the values of the viscoelastic parameters of the monolayer. The present paper deals with a new method of determining the wave parameters. Using small tracer particles, the displacement of the monolayer, as a function of the distance to the oscillating barrier, is made visible and recorded by video cameras. From these recordings the wave parameters and viscoelastic properties are evaluated. A comparison is made between results obtained with this method and those obtained from the measurements of the varying surface tension. This latter method has until now normally been used to determine the wave parameters. Results on cholesterol and decanoic acid monolayers indicate that both methods lead to similar results within experimental error. It is shown that in case of reflected waves the combination of the results of the two methods, as described earlier by Crone $e t$ al., leads to significantly more accurate values of the wave parameters.
\end{abstract}

\section{INTRODUCTION}

In the last decade much attention has been paid to the study of rheological properties of monolayer covered liquid surfaces (1). These properties are important in processes such as emulsification and foaming (food and petroleum technology). They also are of vital importance to the understanding of the surfactant system of the lungs.

Measurements of rheological properties (dilational elasticity and viscosity) can be performed with the aid of the longitudinal surface wave technique as developed by Lucassen and van den Tempel $(2,3)$. In the monolayer a longitudinal wave is generated (in the $x$ direction) by an oscillating barrier (see Fig. 1). This wave is characterized by the propagation characteristics $\kappa$ and $\beta$, i.e., the damping coefficient and the real wave number. These characteristics depend upon the surface rheological properties and can be obtained from measurements of surface pressure $\pi(x, t)$ or surface velocity $v(x, t)$, which both contain the factor $\exp (\beta x-i(\omega t-\kappa x))$ ( $\omega$ is the angular frequency). $\pi(x, t)$ is usually measured with the Wilhelmy-plate technique and $v(x, t)$ can be derived from recording of the displacement of small tracer particles placed on the surface $(8,4)$. Maru and Wasan (8) were the first who reported measurements using tracer particles.

As indicated by Crone et al. (4) simultaneous measurements of $\pi(x, t)$ and $v(x, t)$ give rise to $\kappa$ and $\beta$ values with a higher degree of accuracy than those obtained from measurements of either $\pi(x, t)$ or $v(x, t)$. This is partly due to corrections derived from the simultaneous measurements concerning the imperfect transmission of the barrier motion to the surface.

Crone et al. reported orienting measurements. Their work has been continued since. In particular improvements of the tracer particle technique have been achieved; a video measuring system has been introduced. Results of experiments on cholesterol and decanoic acid monolayers are reported. 


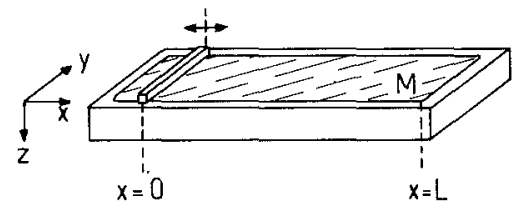

FIG. 1. The Langmuir trough with oscillating barrier at $x=0 . \mathrm{M}$ is the monolayer covered surface.

\section{METHOD}

The tracer particles. As tracer particles we used so-called Eccospheres (obtained from Emerson and Cumming Inc.). These are small hollow glass spheres with diameters ranging from 40 to $100 \mu \mathrm{m}$ and a true density of $300 \mathrm{~kg} \mathrm{~m}^{-3}$. These particles must follow the surface motion without disturbing it. To show this, two effects have to be considered.

First, as a particle is dragged on by the monolayer surrounding it, inertial effects have to be estimated. Therefore, we consider the equation of motion which reads.

$$
m \partial v_{\mathrm{p}} / \partial t=-k\left(v_{\mathrm{p}}-v\right)
$$

where $m$ is the mass of the tracer particle, $k$ the friction coefficient, $v$ the velocity of the monolayer, and $v_{\mathrm{p}}$ the velocity of the tracer particle. For sinusoidal motion Eq. [1] leads to

$$
v_{\mathrm{p}}=v(1 /(1+i \omega \tau))
$$

where $\tau=m / k$. A value for $k$ can be estimated from Stoke's law, taking into account that only one-third of the particle is submerged in the water. For the tracer particles used we find $\tau=10^{-3}$ sec. For the angular frequencies used in our experiments $\omega \tau \ll 1$, thus inertial effects are negligible.

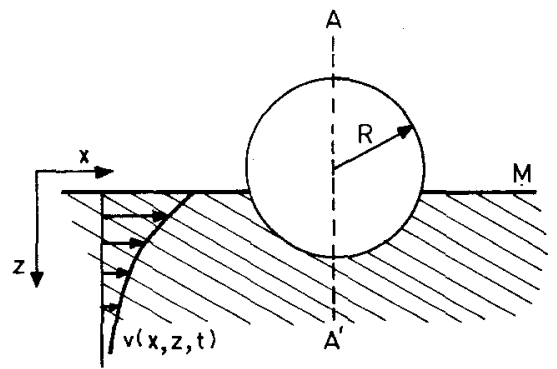

Second, as the particle is partially submerged in the water its amplitude will be somewhat smaller than that of the surface and its movement delayed, due to the exponential velocity profile of $v(x, z, t)$ (see Fig. 2 ), in the formula

$$
\begin{aligned}
v(x, z, t) & =v(x, t) \exp (-n z), \\
n & =(1+i)(\omega \rho / 2 \eta)^{1 / 2} .
\end{aligned}
$$

The velocity $v_{p}(x, t)$ of the tracer particle can be estimated using

$$
\begin{aligned}
v_{\mathrm{p}}(x, t)=\left(1 / A_{\mathrm{t}}\right) \int_{0}^{d} v( & x, t) \\
& \times \exp (-n z) b(z) d z
\end{aligned}
$$

where $d$ is the height of the submerged portion of the tracer particle, $A_{\mathrm{t}}$ the cross-sectional area from that part of the tracer particle (see Fig. 2), and $b(z)$ the width of the tracer particle at height $z$. Using polar coordinates, Eq. [4] can be rewritten as

$$
\begin{aligned}
& v_{\mathrm{p}}(x, t)=\left(1 / A_{\mathrm{t}}\right) \int_{0}^{\alpha_{0}} v(x, t) \\
& \quad \times \exp \left(-n\left(R \cos \alpha-R_{\mathrm{s}}\right)\right) R^{2} \sin ^{2} \alpha d \alpha
\end{aligned}
$$

where $R$ is the radius of the tracer particle, $R_{\mathrm{s}}=R-d$, and $\alpha_{0}=\arccos \left(R_{\mathrm{s}} / R\right)$ (see Fig. 2). From numerical evaluations, corrections concerning amplitude attenuation and phase shift can be calculated. For a tracer particle of average size $(R=35 \mu \mathrm{m})$, an attenuation of $2.5 \%$ and a phase shift of $0.019 \mathrm{rad}$ are thus computed at the highest angular frequency used $\left(\omega=5.24 \mathrm{rad} \mathrm{sec}^{-1}\right)$. Corrections for this effect have been applied throughout.

FIG. 2. A tracer particle (radius $R$ ) in the monolayer covered surface (M). 


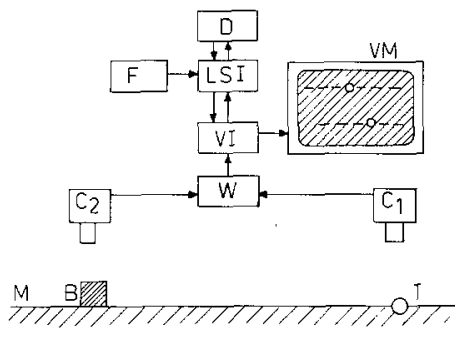

FIG. 3. The experimental set-up. $M$ is the monolayer covered surface, $B$ the barrier, $T$ the tracer particle. $\mathrm{Cl}$ and $C 2$ are video cameras, $W$ the wiper unit, VI the video interface, LSI is the microcomputer with floppy disc (F) and display terminal (D). VM is the video monitor showing two dots representing barrier (upper dot) and tracer particle (lower dot) movement.

Videotechnique. The displacement of the particles is recorded with a video camera $\mathrm{C} 1$ (see Fig. 3). This camera can be positioned at any point above the surface.

In order to obtain the necessary contrast between tracer particle and background, darkfield illumination is applied (5). The displacement of the barrier is recorded with camera $\mathrm{C} 2$. The picture of camera $\mathrm{C} 2$ is inserted in the picture of camera $\mathrm{Cl}$ using a wiper unit (Sony CMW-110CE). This has been done in such a way that the resulting picture on the monitor shows two white dots, moving in parallel to the TV-scan lines, in the upper part and lower part of the screen, respectively (see Fig. 3).

The wiper output is also fed into the VMS (Video Measuring System). The VMS consists of a computer-controlled video interface (developed at the Twente University of Technology (6)) and a DEC LSI-11 microcomputer with floppy disc and display terminal (see Fig. 3). The video-interface discriminates the white dots from the gray background. A computer program, executed by the LSI-11, indicates to the video-interface two picture lines coinciding with the movement of the respective dots. The positions of the dots are measured on these so-called measuring lines. The video-interface interrupts the computer program to fetch the measured positions and to store them on floppy-disc. An off-line pro- gram processes the stored positions, resulting in estimations of amplitudes and phase angles of the movements of both barrier and tracer particle.

The monolayer. A Teflon Langmuir trough $(8 \times 60 \mathrm{~cm})$ was used. Measurements were performed on spread (cholesterol) as well as absorbed (decanoid acid) monolayers. The preparation of the monolayers and measurements of the surface pressure were performed as described earlier (4). The angular frequency of the oscillating barrier could be adjusted to $1.05,2.62$, or $5.24 \mathrm{rad} \mathrm{sec}^{-1}$, and the amplitude was fixed at $0.5 \mathrm{~mm}$ throughout. All experiments were performed at room temperature $\left(21.0 \pm 0.5^{\circ} \mathrm{C}\right)$.

\section{RESULTS AND DISCUSSION}

The amplitude of a traveling wave decreases exponentially with increasing value of $x$. The slope of the logarithmic plot of the amplitude versus $x$ gives the value of $\beta$. The value of $\kappa$ can be obtained from a linear plot
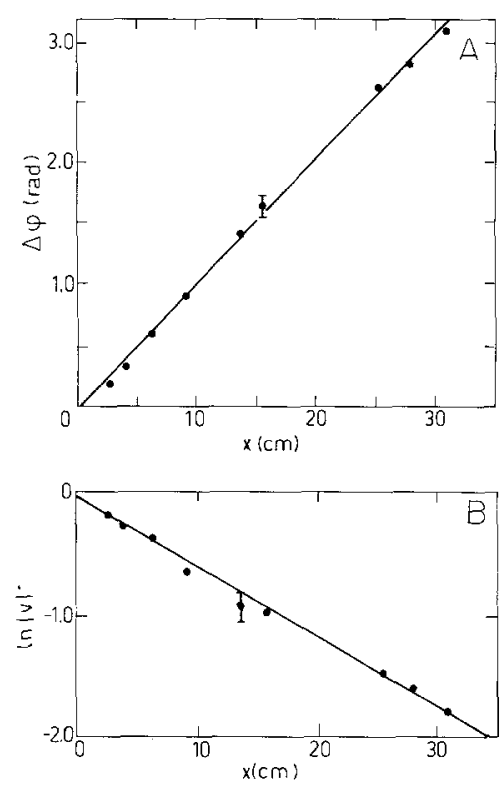

FIG. 4. Phase difference $\Delta \varphi$ (A) and amplitude $\ln |v|$ (B) of the surface velocity wave as a function of $x$, obtained from a decanoic acid monolayer $(\omega=2.62 \mathrm{rad}$ $\sec ^{-1}, \pi=18.10^{-3} \mathrm{~N} \mathrm{~m}^{-1}$ ). 
of the phase difference $(\Delta \phi)$ between the motion of the tracer particle and $x$ and the motion of the barrier at $x=0: \Delta \phi=\kappa \cdot x$.

Figure 4 shows these plots obtained from measurements on a decanoic acid monolayer at $\omega=2.62 \mathrm{rad} \mathrm{sec}-1$. The resulting values for $\kappa$ and $\beta$ from both $v(x, t)$ and $\pi(x, t)$ measurements are listed in Table I, together with those obtained at frequencies $\omega=1.05$ and $5.24 \mathrm{rad} \mathrm{sec}^{-1}$. We conclude that both measurements lead to similar $\kappa$ and $\beta$ values within experimental error. The dispersion equation (4), $i\left(\epsilon_{\mathrm{d}}+i \omega \eta_{\mathrm{d}}\right)(\kappa-i \beta)^{2}=\eta \omega n$, shows the relation between $\kappa, \beta$, and the rheological properties of the monolayer, that is, surface dilational elasticity $\epsilon_{\mathrm{d}}$ and surface dilational viscosity $\eta_{\mathrm{d}}$. Using the mean $\kappa$ and $\beta$ values from the $v(x, t)$ and the $\pi(x, t)$ measurement, the values of $\epsilon_{\mathrm{d}}$ and $\eta_{\mathrm{d}}$ are calculated as listed in Table III. These results are in good agreement with those reported by Lucassen and van den Tempel $(2,3)$. Figure 5 gives a typical plot of the amplitude and phase versus $x$ of $v(x, t)$ for a cholesterol monolayer a $\omega=5.24 \mathrm{rad} \mathrm{sec}^{-1}$. From the measured points in Fig. 5B it is clear that we are not dealing with a single traveling wave (no exponential decay). Due to reflections at the end of the trough $(x=L)$ we are concerned with two waves, one traveling in the positive and the other in the negative $x$-direction. This gives the following expression for $v(x, t)$

\section{TABLE I}

$\kappa$ and $\beta$ Values (and Their Experimental Errors) Obtained from a Decanoic Acid Monolayer $\left(\pi=18 \times 10^{-3}\right.$ $\mathrm{N} \mathrm{m}^{-1}$ ) at Various Angular Frequencies

\begin{tabular}{ccc}
\hline $\begin{array}{c}\text { Angular frequency } \\
\left(\text { rad } \mathrm{sec}^{-1}\right)\end{array}$ & $\begin{array}{c}\text { Tracer particle method } \\
\left(\mathrm{m}^{-1}\right)\end{array}$ & $\begin{array}{c}\text { Wilhelmy plate method } \\
\left(\mathrm{m}^{-1}\right)\end{array}$ \\
\hline \multirow{2}{*}{1.05} & $\beta=3.2 \pm 0.2$ & $2.7 \pm 0.3$ \\
& $\kappa=5.6 \pm 0.4$ & $5.6 \pm 0.6$ \\
& & \\
2.62 & $\beta=5.3 \pm 0.2$ & $5.6 \pm 0.2$ \\
& $\kappa=10.2 \pm 0.5$ & $10.1 \pm 0.5$ \\
5.24 & $\beta=7.6 \pm 0.8$ & $7.4 \pm 0.8$ \\
& $\kappa=15 \pm 1$ & $15 \pm 2$ \\
\hline
\end{tabular}

\section{TABLE II}

$\kappa$ and $\beta$ Values (and Their Experimental Errors) Obtained from a Cholesterol Monolayer $\left(\pi=9 \times 10^{-3} \mathrm{~N}\right.$ $\mathrm{m}^{-1}$ ) at Various Angular Frequencies

\begin{tabular}{lccc}
\hline $\begin{array}{c}\text { Angular } \\
\text { frequency } \\
(\text { rad sec }\end{array}$ & $\begin{array}{c}\text { Tracer particle } \\
\text { method } \\
\left(\mathrm{m}^{-1}\right)\end{array}$ & $\begin{array}{c}\text { Wilhelmy plate } \\
\text { method } \\
\left(\mathrm{m}^{-1}\right)\end{array}$ & $\begin{array}{c}\text { Corrected } \\
\text { values } \\
\left(\mathrm{m}^{-1}\right)\end{array}$ \\
\hline 1.05 & $\beta=0.6 \pm 0.1$ & $0.6 \pm 0.2$ & $0.48 \pm 0.03$ \\
& $\kappa=1.2 \pm 0.3$ & $1.0 \pm 0.2$ & $1.08 \pm 0.03$ \\
2.62 & $\beta=0.9 \pm 0.1$ & $1.0 \pm 0.2$ & $0.95 \pm 0.05$ \\
& $\kappa=2.2 \pm 0.2$ & $2.0 \pm 0.2$ & $2.15 \pm 0.05$ \\
5.24 & $\beta=1.7 \pm 0.1$ & $1.6 \pm 0.2$ & $1.50 \pm 0.05$ \\
& $\kappa=3.6 \pm 0.1$ & $3.5 \pm 0.2$ & $3.6 \pm 0.1$ \\
\hline
\end{tabular}

$$
v(x, t)=v_{0}\left\{\frac{e^{-i k(x-L)}-e^{i k(x-L)}}{e^{i k L}-e^{-i k L}}\right\} e^{i \omega t}
$$

where $v_{0}$ is the velocity at the origin (equal to the velocity $v_{\mathrm{b}}$ of the barrier) and $k=\kappa$ $-i \beta$.

For $\pi(x, t)$ we now have

$$
\pi(x, t)=\frac{k \in v_{0}}{\omega}\left\{\frac{e^{-i k(x-L)}+e^{i k(x-L)}}{e^{i k L}-e^{-i k L}}\right\} e^{i \omega t}
$$

where $\epsilon$ stands for the complex surface elasticity $\epsilon=\epsilon_{\mathrm{d}}+i \omega \eta_{\mathrm{d}}$. Equations [6] and [7] enable the values of $\kappa$ and $\beta$ to be determined from the measured values of amplitude and phase of $v(x, t)$ and $\pi(x, t)$. These values and those obtained at $\omega=2.62$ and $1.05 \mathrm{rad} \mathrm{sec}^{-1}$ are presented in Table II. We conclude that for cholesterol monolayers the $v(x, t)$ and $\pi(x, t)$ measurements give the same $\kappa$ and $\beta$ values (within experimental error). By introducing into Eq. [6] the $\kappa$ and $\beta$ values obtained from the $v(x, t)$ measurement we obtain the curves presented as continuous lines in Fig. 5. We note that near $x=0$ (Fig. 5B) there is a significant deviation between theory and experimental values. This phenomenon was observed generally in our experiments and has also been reported by Crone et al. (4). The discrepancy can be explained by assuming that the transmission of the barrier motion to the monolayer is not perfect, hence in Eqs. [6] and [7] $v_{0}$ is not equal to $v_{\mathrm{b}}$. In order to overcome this, a new fitting 
TABLE III

Dilational Elasticity and Viscosity (and Their Experimental Errors) Obtained from Cholesterol ( $\pi=9 \times 10^{-3}$ $\left.\mathrm{N} \mathrm{m}^{-1}\right)$ and Decanoic Acid $\left(\pi=18 \times 10^{-3} \mathrm{~N} \mathrm{~m}^{-1}\right)$ Monolayers at Various Angular Frequencies

\begin{tabular}{cccccc}
\hline & \multicolumn{2}{c}{ Cholesterol } & & \multicolumn{2}{c}{ Decanoic acid } \\
\cline { 2 - 3 } \cline { 5 - 6 } $\begin{array}{c}\text { Angular frequency } \\
(\text { rad sec }\end{array}$ & $\begin{array}{c}\text { Dilational elasticity } \\
\left(\mathrm{N} \mathrm{m}^{-1}\right)\end{array}$ & $\begin{array}{c}\text { Dilational viscosity } \\
\left(\mathrm{Nsec} \mathrm{m}^{-1}\right)\end{array}$ & & $\begin{array}{c}\text { Dilational elasticity } \\
\left(\mathrm{N} \mathrm{m}^{-1}\right)\end{array}$ & $\begin{array}{c}\text { Dilational viscosity } \\
\left(\mathrm{Nsec} \mathrm{m}^{-1}\right)\end{array}$ \\
\hline 1.05 & $0.77 \pm 0.02$ & $0.04 \pm 0.03$ & & $0.027 \pm 0.002$ & $0.006 \pm 0.002$ \\
2.62 & $0.77 \pm 0.02$ & $0.01 \pm 0.01$ & & $0.033 \pm 0.002$ & $0.003 \pm 0.001$ \\
5.24 & $0.79 \pm 0.03$ & $0.00 \pm 0.005$ & & $0.045 \pm 0.002$ & $0.0014 \pm 0.0004$ \\
\hline
\end{tabular}

procedure has been applied in which $v_{0}$ is not taken equal to $v_{\mathrm{b}}$. To obtain the most accurate values possible, both $v(x, t)$ and $\pi(x, t)$ measurements have been fitted simultaneously. The new $\kappa$ and $\beta$ values are also listed in Table II as "corrected values." From the best fits $v_{0}$ is calculated, we found
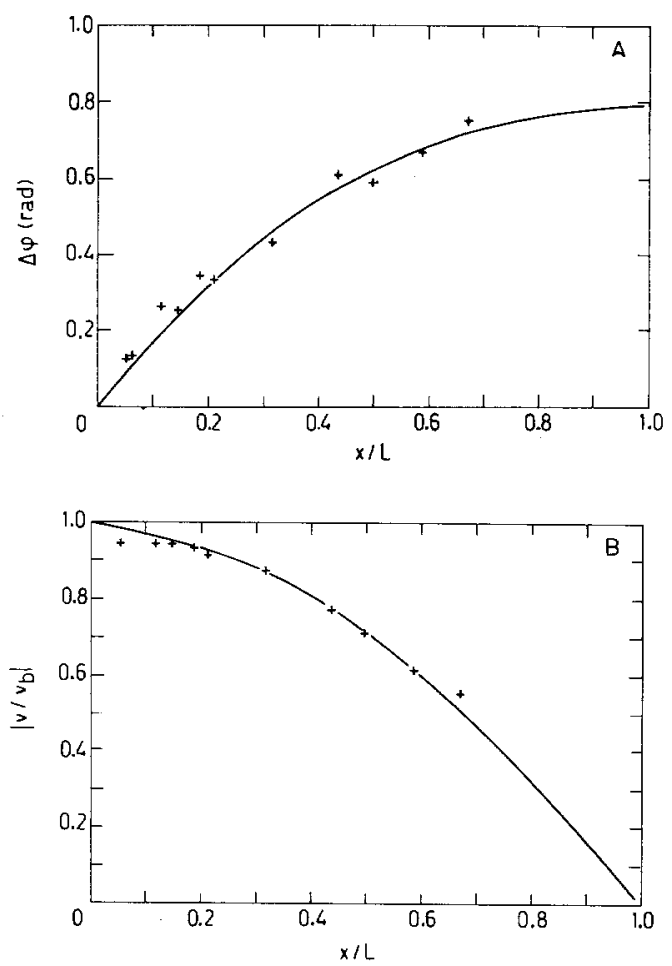

FIG. 5. Phase difference $\Delta \varphi$ (A) and (dimensionless) amplitude $\left|v / v_{\mathrm{b}}\right|(\mathrm{B})$ of the surface velocity wave as a function of the (dimensionless) distance $x / L$, obtained from a cholesterol monolayer $\left(\omega=5.24 \mathrm{rad} \mathrm{sec}^{-1}\right.$, $\pi=9 \times 10^{-3} \mathrm{~N} \mathrm{~m}^{-1}$ ). that $\left|v_{0}\right|=0.95\left|v_{\mathrm{b}}\right|$ throughout and $\Delta \varphi_{v_{0}}$ $=\Delta \varphi_{v_{\mathrm{b}}}-0.02$ at $\omega=1.05 \mathrm{rad} \sec ^{-1}$. At $\omega$ $=2.62$ and $5.24 \mathrm{rad} \mathrm{sec}^{-1}$ no phase shift was found $\left(\Delta \varphi_{v_{0}}=\Delta \varphi_{v_{\mathrm{b}}}\right)$.

We learn from Table II that the effect of the imperfect barrier transmission on the results is too significant to be neglected (for instance see the $\beta$ value at $\omega=1.05 \mathrm{rad} \mathrm{sec}^{-1}$ ). In Table III, $\epsilon_{\mathrm{d}}$ and $\eta_{\mathrm{d}}$ values are given as calculated from the "corrected" $\kappa$ and $\beta$ values.

When we compare our set-up with that described by Maru and Wasan (8) we note that the use of the video system leads to greater accuracy, the whole particle motion instead of its extreme positions is used to calculate the amplitude and phase of $v(x, t)$. From previous experiments (4) with handclocking (as done by Maru) we estimate that, for $\omega=1.05 \mathrm{rad} \mathrm{sec}^{-1}$, the video method leads to at least 10 times greater an accuracy for $\Delta \varphi_{v}$.

It might be of interest to apply the corrections for the imperfect barrier transmission to the results of Maru. As the discrepancies between theory and experimental values (for $x$ near zero) are rather pronounced in Maru's results, these corrections might influence his conclusion as to the discrepancy between his results from $\pi(x, t)$ and $v(x, t)$ measurements.

In the previous results, the measurement of the wave was done by means of tracer particles and only those particles which were more than $2 \mathrm{~cm}$ away from the side walls were recorded. This was done in order to minimize the influence of surface shear 
caused by the side walls (7). Experiments were done to see if the surface shear had influence on the measurement under this experimental condition. For this purpose, the amplitude of $v(x, t)$ was checked, at constant $x$ value, and expressed as a function of $y$ (Fig. 1). The amplitude remained constant proving that surface shear had no influence. This conclusion proved to hold for experiments on cholesterol as well as decanoic acid monolayers.

From the present results we conclude that measurement of $v(x, t)$ has proved to be useful in the study of rheological properties of monolayers. Compared with Crone et al. (4) the accuracy of the measurement of $v(x, t)$ has improved markedly.

\section{REFERENCES}

1. van den Tempel, M., J. Non-Newtonian Fluid Mech. 2, 205 (1977).

2. Lucassen, J., and van den Tempel, M., J. Colloid Interface Sci. 41, 401 (1972).

3. Lucassen, J., and van den Tempel, M., Chem. Eng. Sci. 27, 1283 (1972).

4. Crone, A. H. M., Snik, A. F. M., Poulis, J. A., Kruger, A. J., and van den Tempel, M., J. Colloid Interface Sci. 74, 1 (1980).

5. Levi, L., “Applied Optics," p. 475. John Wiley, New York, 1960.

6. Houkes, Z., "IFAC Digital Computer Applications to Process Control," p. 231. Pergamon Press, Oxford, 1981.

7. De Feyter, J. A., J. Colloid Interface Sci. 69, 375 (1979).

8. Maru, H. C., and Wasan, D. T., Chem. Eng. Sci. 34, 1295 (1979). 